\title{
LA-UR-21-25486
}

Approved for public release; distribution is unlimited.

Title: $\quad$ Conducting A Literature Review \& Writing an Introduction

Author(s): $\quad$ Cummings, lan Thomas

Intended for: Distribute to students in LADSS program

Issued: 2021-06-10 
Disclaimer:

Los Alamos National Laboratory, an affirmative action/equal opportunity employer, is operated by Triad National Security, LLC for the National Nuclear Security Administration of U.S. Department of Energy under contract 89233218CNA000001. By approving this article, the publisher recognizes that the U.S. Government retains nonexclusive, royalty-free license to publish or reproduce the published form of this contribution, or to allow others to do so, for U.S. Government purposes. Los Alamos National Laboratory requests that the publisher identify this article as work performed under the auspices of the U.S. Department of Energy. Los Alamos National Laboratory strongly supports academic freedom and a researcher's right to publish; as an institution, however, the Laboratory does not endorse the viewpoint of a publication or guarantee its technical correctness. 


\section{Conducting A Literature Review \& Writing an Introduction}

lan T. Cummings, Ph.D.

itc@lanl.gov

Space \& Remote Sensing Group \& Engineering Institute

June 9, 2021 


\section{Who Am I?}

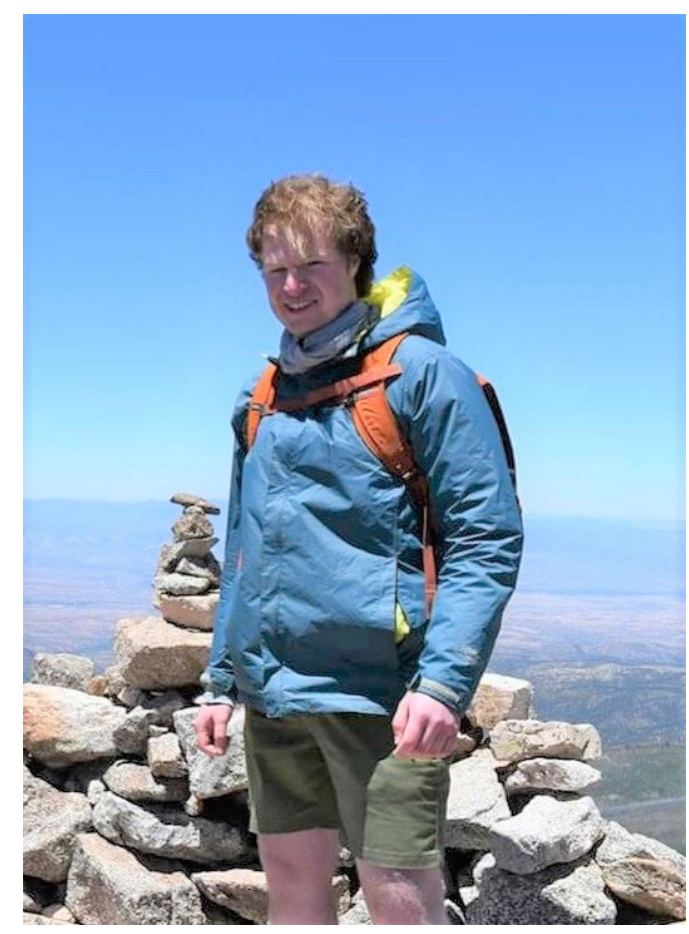

Education - Michigan Technological University

BS \& MS in Computer Engineering

Ph.D. in Electrical Engineering

NSF GRFP Recipient

\section{Experience}

LADSS 2015 Alumnus

MIT Lincoln Laboratory Summer Research Intern LANL Postdoc 2020-Present Ultrasonic NDE Research 


\section{Outline}

- Introductions (The Person-to-Person Kind)

- Introductions (The Technical Paper Kind)

- What are they supposed to accomplish?

- Mapping a path from idea to words on paper

- Common pitfalls \& how to avoid/handle them

- Ways to make your life easier

- Live Demo! 


\section{Survey of Experience!}




\section{What is an introduction supposed to do?}

1. Introduce \& motivate the problem

2. Lit Review

- Describe the current state of the art (work by others)

- Describe your (team's/mentors'?) previous related work

3. Set the work described in this paper apart (describe the novelty)

4. Describe the structure of the paper to come (?)

- Honestly this one's a little take-it-or-leave-it for me

5. Notation (?)

- Only need this if your paper get's pretty math-heavy

- Typically:

- In this order

- 1-2 pages total 


\section{Charting a Path from Idea to Paper}

Take a reader from what they know to what you need them to know

- This requires:

- Understanding who your audience is/what you can expect them to know

- Understanding specifically what you need them to know

- Pro-Tip: write as if you're explaining it to your past self just starting the project 


\section{Charting a Path from Idea to Paper}

\section{Introduce \& Motivate the Problem}

- Step 1: Figure out what you need them to know

- Draft a few sentences describing the work that you're going to do (but write it in the past tense...)

- This statement will evolve during the lit review \& the research, that's normal

- Step 2: Figure out what you can expect them to be familiar with

- Your audience for IMAC:

- Undergrad/Grad students like yourself

- Chuck, Pete Avitable

- Everything in between

- Step 3: Start writing!

- 1-2 Paragraphs

- Start by discussing the broad topic/capability gap/pain point

- e.g. The dynamics of big buildings are hard/expensive/critical to monitor...

- Narrow down toward the specific technical problem

- e.g. Video based motion magnification offers a simple measurement solution, but it introduces [difficulty] in terms of data processing... 


\section{Charting a Path from Idea to Paper}

\section{Lit Review}

- Step 1: Identify relevant search terms, publications, timeframe

- Goal is to figure out how others have tried to solve this/similar problem

- Step 2: Take those parameters to Google Scholar/[Insert Your Favorite Database]

- Open a new tab for each article with a relevant title

- Down-select for relevance again by reading the abstracts

- Save the PDF \& citation information (a citation manager is helpful here)

- Step 3: Read \& Take Notes!

- Start reading each paper

- In the introduction of the paper, make a short bulleted list (2-4) for each paper you read

- Highlight the big/important/unique features of the paper only

- Avoid repeated/common information

- Use your own words, plagiarism is bad

- If you're using an automated citation generator, include the citation tag/reference now

- Step 4: Arrange the sources into coherent groups (e.g. similar approaches)

- Step 5: Condense the lists into prose

- Team's Related work section is a similar but simpler process of describing the relevant aspects of previous work 


\section{Charting a Path from Idea to Paper}

\section{Describe the Novelty of Your Work}

- 1-2 Paragraphs

- Identify aspects of your work that are different/more general/more advanced than previous works (others \& yours)

\section{Summary of Paper to Come}

- 1 Paragraph

- A sentence describing what each section will say

- "Section 2 describes the theoretical background of this work, including the operating principles of a Turboencabulator."

\section{Notation}

- 1 Paragraph

- "Lowercase symbols refer to scalars, bold lowercase symbols refer to column vectors, bold uppercase symbols refer to matrices, $(\cdot)^{H}$ represents Hermitian transposition..." 


\section{Common Pitfalls \& How to Avoid/Handle Them}

- "What if I miss a relevant paper in the lit review?"

- Honestly not a big deal

- If a reviewer brings one to your attention, you can add it in

- "What if I found a paper that does exactly what we were planning to do?"

- It's highly unlikely that the paper actually does exactly what you proposed

- There are typically caveats/restrictions to their work that you could address (may have to dig into the paper to find them)

- Work with your teammates \& mentors to find the novel aspects of your work

" "This is taking FOREVER...."

- Yeah, it might feel that way, but it's an important step

- No this is not some research hazing exercise

- I promise it gets better/easier/faster with practice

- Literature Review part of introduction turns into a list of "Bla et al. did bla bla bla [1]."

- Group similar sources and describe the overall direction \& important aspects of the research

- Avoid repeating the common aspects. Mention them once and use paragraphs/subheadings to imply common elements

- Be intentional about varying sentence structure 


\section{Making Your Life Easier}

- Use a citation manager

- EndNote, Zotero, etc.

- Automatically:

- Downloads citation information (sometimes this is a little finicky)

- Formats citations in your desired style

- Generates Bibliography in Word

- Exports to BibTex (for LaTeX, again sometimes this is a little finicky)

- Be careful about LANL information security/cloud services policies

- Set reasonable limits on how wide you cast your net for sources

- What is still relevant? (Date)

- Restrict yourself to more reputable publications when possible

- Start writing the introduction in the final paper template (less reformatting work later) 


\section{Live Demo! \\ To the Interwebs!}

